\title{
Comportamento da dor e da função pulmonar em pacientes submetidos à cirurgia cardíaca via
} esternotomia

\author{
Pain and pulmonary function in patients submitted to heart surgery via sternotomy
}

\author{
Maria Cristina dos Santos BAUMGARTEN ${ }^{1}$, Gisele Kalata GARCIA², Michele Hagi FRANTZESKI ${ }^{3}$, Cristiane
} Mecca GIACOMAZZI ${ }^{4}$, Verlaine Balzan LAGNI ${ }^{5}$, Alexandre Simões DIAS ${ }^{6}$, Mariane Borba MONTEIRO ${ }^{7}$

RBCCV 44205-1124

\section{Resumo}

Objetivo: Avaliar o comportamento da função pulmonar e da dor em pacientes adultos submetidos à cirurgia cardíaca por esternotomia. Além de verificar possíveis correlações e comparações dessas variáveis com as características do procedimento cirúrgico e o tempo de internação hospitalar.

Métodos: Foi realizado estudo de coorte composto de 70 indivíduos, nos quais foi avaliada a função pulmonar préoperatória por espirometria e inspirometria de incentivo. Os pacientes foram acompanhados no pós-operatório, por meio de protocolo com informações da cirurgia, função pulmonar e um protocolo de avaliação álgica (escala análoga visual e desenho do corpo humano).

Resultados: Os valores de função pulmonar do período pós-operatório apresentaram diminuição significativa em relação ao pré-operatório $(P<\mathbf{0 , 0 1})$. A dor localizou-se na região da esternotomia, persistindo até o $5^{\circ}$ dia de pósoperatório. Houve correlação da dor com os parâmetros de função pulmonar (volume expiratório forçado no $1^{\circ}$ segundo - percentual $r=-0,271$ e $P<0,047$; pico de fluxo expiratório

1. Fisioterapeuta; Pós-graduanda em Fisioterapia Cardiorrespiratória pelo Instituto de Cardiologia do Rio Grande do Sul.

2. Fisioterapeuta; Instrutora de Pilates.

3. Fisioterapeuta; Docente do Curso de Fisioterapia do Centro Universitário Metodista do Sul IPA, Pós-graduanda em Fisioterapia em Pediatria e Neonatologia pelo Hospital Moinhos de Vento de Porto Alegre, Rio Grande do Sul.

4. Fisioterapeuta; Residente em Reabilitação Cardiopulmonar do Programa de Residência Multidisciplinar em Saúde da Pontifícia Universidade Católica do Rio Grande do Sul (PREMUS PUCRS).

5. Fisioterapeuta, Especialista em Cinesiologia pela Universidade Federal do Rio Grande do Sul (UFRGS); Docente do Curso de Fisioterapia do Centro Universitário Metodista do Sul IPA.

6. Fisioterapeuta, Doutor em Fisiologia pela UFRGS; Docente do Curso de Fisioterapia do Centro Universitário Metodista do Sul IPA. $r=-0,357$ e $P<0,008$; volume inspiratório máximo $r=-0,293$ e $P<0,032)$. Não se observou correlação significativa da dor com outras variáveis.

Conclusão: Observou-se prejuízo significativo da função pulmonar, não se restabelecendo completamente até o $5^{\circ}$ dia de pós-operatório. A dor foi uma queixa que persistiu durante todo o período do estudo. Os parâmetros de função pulmonar apresentaram relação significativa com a dor. Não houve correlação entre dor e as características dos indivíduos, do procedimento cirúrgico e tempo de internação hospitalar.

Descritores: Complicações pós-operatórias. Testes de função respiratória. Dor pós-operatória. Medição da dor.

\section{Abstract}

Objective: To investigate the pulmonary function and pain in adult patients undergoing heart surgery via sternotomy and to verify possible correlations of these variables with the characteristics of the surgical procedure and hospital stay.

Methods: A cross-sectional study was carried out of 70

7. Fisioterapeuta, Doutora em Ciências Pneumológicas pela UFRGS; Docente do Curso de Fisioterapia do Centro Universitário Metodista do Sul IPA.

Trabalho realizado no Centro Universitário Metodista do Sul IPA Porto Alegre, RS, Brasil.

Endereço para correspondência

Maria Cristina dos Santos Baumgarten. Rua Tito Lívio Zambecari, 216/304 - Mon't Serrat - Porto Alegre, RS, Brasil - CEP: 90450230

E-mail: crisbaumg@terra.com.br

Artigo recebido em 5 de junho de 2009 Artigo aprovado em 13 de novembro de 2009 
individuals undergoing heart surgery. The lung function was assessed before and after surgery by spirometry and incentive spirometry. Details of the surgical procedure were studied and patients were followed up postoperatively using a visual analogue scale and design of the human body to evaluate pain.

Results: The pulmonary function was significantly impaired in the postoperative compared to preoperative period $(P<0.01)$. The pain was centered in the region of the sternotomy and persisted until at least the $5^{\text {th }}$ postoperative day. There was a correlation between pain and the parameters of pulmonary function (forced expiratory volume in 1 second - percentage: $r=\mathbf{- 0 . 2 7 1}, P<0.047$; peak expiratory flow: $r=$ $0.357, P<0.008$; and maximum inspiratory volume: $r=-0.293$,

\section{INTRODUÇÃO}

A dor é a principal manifestação relatada por pacientes submetidos à cirurgia cardíaca, apresentando caráter multifatorial. Os fatores que influenciam na dor podem ser: incisão cirúrgica, retração e dissecção tecidual durante o procedimento cirúrgico, múltiplas canulações intravenosas, drenos torácicos e procedimentos invasivos que esses pacientes são submetidos durante o regime terapêutico [1].

A esternotomia mediana longitudinal é a abordagem mais usada para as cirurgias cardíacas, devido à exposição da região. Entretanto, essa abordagem pode alterar significativamente a função pulmonar pela consequente instabilidade do tórax superior. Segundo a literatura, o quadro álgico originado pelos procedimentos da rotina cirúrgica associado ao grande estímulo nociceptivo da esternotomia torna-se fonte importante para a morbidade e mortalidade, interferindo na evolução do pós-operatório (PO). A técnica cirúrgica pode alterar as trocas gasosas, modificando a mecânica ventilatória e gerando alterações restritivas da função pulmonar [2-5].

Westerdahl et al. [6] relatam que indivíduos após a cirurgia de revascularização do miocárdio (CRM) podem apresentar atelectasias, redução importante dos volumes pulmonares e hipoxemia no PO imediato. Essas complicações podem estar relacionadas aos efeitos da anestesia, aos eventos no transoperatório, à disfunção diafragmática, à medicação e ao estado hemodinâmico desses pacientes [7].

Na literatura, a dor PO é descrita como sendo um dos fatores responsáveis pela disfunção respiratória associado a outros fatores descritos anteriormente [8,9]. A intensidade desse sintoma aumenta principalmente quando é feita a manipulação da pleura parietal para realizar o enxerto da artéria torácica interna esquerda (ATIE), sendo responsável por um trauma cirúrgico adicional e redução de força muscular inspiratória [10]. Por isso, o conceito de que a dor
$P<0.032)$. There was no significant correlation between pain and other variables.

Conclusion: There was significant impairment of lung function which had not recovered completely on the $5^{\text {th }}$ postoperative day. Pain was a complaint that persisted throughout the study period. The parameters of pulmonary function showed a significant relationship with pain. There was no correlation between pain and the characteristics of individuals, the surgical procedure or the length of hospital stay.

Descriptors: Postoperative complications. Respiratory function tests. Pain, postoperative. Pain measurement.

PO é normal e esperada, associado à falta de conhecimento da fisiologia da dor e da farmacologia dos analgésicos, faz com que a atenção da equipe esteja voltada às complicações pós-operatórias mais comuns do que ao sintoma que mais incomoda o paciente: a dor [11]. Conhecer melhor as características da dor no PO e sua influência na função pulmonar durante este período pode ser o início para o desenvolvimento de estratégias para melhorar seu controle.

O objetivo desse estudo foi avaliar o comportamento da função pulmonar e da dor em pacientes adultos submetidos à cirurgia cardíaca por esternotomia, além de verificar possíveis correlações e comparações dessas variáveis com as características do procedimento cirúrgico e o tempo de internação hospitalar.

\section{MÉTODOS}

A pesquisa caracteriza-se por ser um estudo de coorte prospectivo, com amostra não-probabilística, intencional, realizado no período de março de 2005 a setembro de 2007, no Complexo Hospitalar Santa Casa (CHSC). O estudo foi submetido e aprovado pelos comitês de Ética e Pesquisa das Instituições envolvidas (Centro Universitário Metodista do Sul IPA e CHSC), sob números de protocolo 1142/04 e 930/04, respectivamente.

Os pacientes incluídos nesse estudo preencheram os seguintes critérios de inclusão: idade superior a 18 anos, ambos os sexos, sem história prévia de cirurgia torácica e que realizariam cirurgia cardíaca via esternotomia. Os pacientes foram selecionados a partir da lista de internação do Sistema Único de Saúde (SUS) da Unidade de Cardiologia da Policlínica Santa Clara. Todos participantes foram orientados quanto ao estudo e assinaram previamente o termo de consentimento livre e esclarecido (TCLE).

Foram considerados critérios de exclusão: angina instável Classe III (Classificação de Braunwald), cirurgia 
em caráter de urgência ou emergência e tumor mediastinal. Inicialmente foram selecionados 125 pacientes para o estudo. Destes, 27 foram excluídos; sendo que 33,3\% dos pacientes se recusaram a participar do estudo.

Participaram do estudo 98 pacientes, porém, 28 deles não completaram todas as etapas, sendo consideradas perdas do estudo. Os principais motivos foram: cirurgias antecipadas ou canceladas, datas de procedimentos cirúrgicos posteriores ao período de colheita dos dados, não realização de todos os testes propostos por queixa de cansaço e tempo de intubação superior a 24 horas (Figura 1).

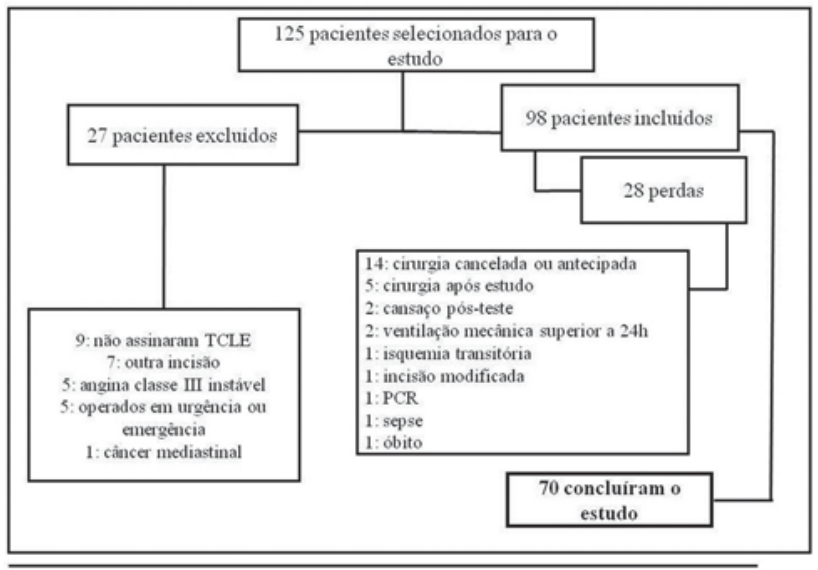

Legenda: TCLE - termo de consentimento livre e esclarecido; $\mathrm{PCR}$ - parada cardiorrespiratória

Fig.1 - Dados dos pacientes relacionados para o estudo

A pesquisa realizou-se com 70 indivíduos submetidos, eletivamente, à CRM e/ou implante de prótese valvar (metálica ou biológica) por esternotomia, com ou sem uso de circulação extracorpórea (CEC), e que foram extubados de acordo com a rotina do serviço.

Durante o período pré-operatório foi realizada uma avaliação que consistia na colheita dos dados de identificação do paciente, informações detalhadas sobre os fatores de risco e doenças associadas. Constava também a avaliação da função pulmonar por meio de espirometria e inspirometria de incentivo (para obtenção estimada do volume inspiratório máximo) [12,13].

A função pulmonar, avaliada por meio dos espirômetros das marcas Micro Medical Limited ${ }^{\circledR}$, modelo MicroPlus, de Kent (England) e Vitalograph ${ }^{\circledR}$, modelo Vitalograph micro, de Ennis (Ireland), forneceu a capacidade vital forçada (CVF), o volume expiratório forçado no primeiro segundo $\left(\mathrm{VEF}_{1}\right)$, o coeficiente expiratório forçado no primeiro segundo $\left(\mathrm{VEF}_{1} /\right.$ CVF\%) e o pico de fluxo expiratório (PFE). O paciente, 15 minutos após a manobra espirométrica, foi instruído a realizar a inspirometria de incentivo a partir do equipamento da marca Sherwood, modelo Voldyne ${ }^{\circledR}$, escolhido pelo baixo custo e fácil reprodutibilidade do método para obtenção do volume inspiratório máximo (Vimáx) [12,13]. Para ambos os testes foram realizadas três manobras intercaladas por um minuto de repouso. A espirometria seguiu os critérios estabelecidos pelas Diretrizes para Testes de Função Pulmonar [14]. A melhor das três manobras foi adotada, contanto que apresentasse diferença menor do que $10 \%$ do segundo maior valor obtido.

Todos os pacientes saíram do bloco cirúrgico com o dreno ${ }^{\circ} 36$ (marca Braile ${ }^{\circledR}$, São Paulo, SP, Brasil), com localização pleural em 13 (18,57\%) pacientes, mediastinal em 19 (27,14\%) e em ambas 38 (54,28\%). Os pacientes foram acompanhados no $1^{\circ}, 2^{\circ}, 3^{\circ}$ e $5^{\circ}$ PO. Durante esse período, utilizou-se um protocolo de acompanhamento com informações das características do procedimento cirúrgico e dados da função pulmonar. Para avaliação da dor utilizouse um protocolo específico, que consistia em um desenho do corpo humano, com vista anterior e posterior, dividido em 37 áreas anatômicas. O indivíduo foi instruído a marcar a área mais álgica, com concomitante aplicação de uma escala subjetiva análogo visual (EAV), de 0 a 10 centímetros, com 0 representando nenhuma dor e 10 como a dor mais intensa para aquela área (protocolo de avaliação da dor utilizado por Mueller et al.) [1]. Ao final do período de acompanhamento foi verificado o tempo de internação hospitalar dos pacientes.

No período de PO imediato, a analgesia utilizada pelo serviço consistia em uso de morfina (2 a 10mg), lentamente, diluída em $9 \mathrm{ml}$ de água destilada acrescida de $1 \mathrm{ml}$ de morfina a cada 3 ou 4 horas, sendo a infusão contínua de 2 a 5mg por hora (quando necessário). Durante a permanência hospitalar foi utilizado paracetamol 750mg ou 500mg de 6/ 6h (se necessário). As avaliações antes e após a cirurgia foram realizadas de forma padronizada pelos autores, com controle do uso dos analgésicos.

O programa estatístico utilizado para a análise dos dados foi o Statistical Package for the Social Sciences (SPSS) versão 17.0. Inicialmente, foi realizado o teste de normalidade, Kolmogorov-Smirnov, para verificar se as variáveis contínuas tinham distribuição paramétrica. Na análise descritiva, as variáveis contínuas foram expressas por média, mediana, intervalos interquartis 25-75 e desvio padrão, e as variáveis categóricas por frequência e porcentual. Na comparação das provas de função pulmonar no pré e pós-operatório, utilizou-se o teste de análise de variância ANOVA, com ajuste de Bonferroni. Para comparação da dor nos dias de pós-operatório foi utilizado o teste de Friedman, seguido do teste de Wilcoxon, com ajuste de Finner. Na comparação do tipo de cirurgia com as variáveis de função pulmonar e dor, utilizou-se o teste não paramétrico Mann Whitney. O coeficiente de correlação de Spearman foi utilizado para apresentar a correlação entre duas variáveis contínuas. O nível de significância adotado foi de $P<0,05$. 


\section{RESULTADOS}

As características dos indivíduos e dos procedimentos cirúrgicos, bem como os principais fatores de risco para complicações pós-operatórias e o tempo de internação hospitalar estão apresentadas na Tabela 1.

Os procedimentos cirúrgicos realizados foram a troca valvar em 34 (48,57\%) pacientes, a CRM em 27 (38,57\%) e a cirurgia combinada em nove (troca de valva associada à CRM - 12,85\%). Em relação a CRM, em 14 (20\%) pacientes foi utilizada a veia safena e, em 13 (18,57\%), a veia safena e a ATIE. Na cirurgia de troca valvar, 13 $(18,57 \%)$ pacientes foram submetidos a substituição por prótese metálica aórtica e $11(15,71 \%)$ por prótese biológica aórtica. O tipo de cirurgia combinada mais frequente foi a CRM utilizando a veia safena associada à troca valvar substituída por prótese biológica aórtica em três (4,28\%) pacientes.

O comportamento da função pulmonar do período pósoperatório em relação ao pré-operatório está demonstrado na Tabela 2. No $1^{\circ}$ pós-operatório, os valores diminuíram significativamente em relação ao pré-operatório para as variáveis $\mathrm{VEF}_{1}$, CVF, PFE e Vimáx. Até o $5^{\circ}$ pós-operatório esses valores não haviam retornado aos anteriores à cirurgia.
Tabela 1. Características dos pacientes, fatores de risco para complicações pós- operatórias e características dos procedimentos cirúrgicos.

\begin{tabular}{|c|c|}
\hline Variáveis & Total $\mathrm{n}=70$ \\
\hline Sexo masculino $\mathrm{n}(\%)$ & $45(64,3 \%)$ \\
\hline Idade (anos) * & $56,72 \pm 12,74$ \\
\hline IMC $(\mathrm{kg} / \mathrm{m} 2) *$ & $26,04 \pm 4,67$ \\
\hline $\mathrm{FE}(\%) *$ & $57,58 \pm 13,37 \%$ \\
\hline Presença de tabagismo n (\%) & $15(21,4 \%)$ \\
\hline Cigarros por dia* & $26,18 \pm 16,09$ \\
\hline Tempo de tabagismo (anos) * & $22,95 \pm 12,8$ \\
\hline Fatores de risco cardiovascular n (\%) & $54(74,3 \%)$ \\
\hline Hipertensão arterial & $45(64,2 \%)$ \\
\hline Dislipidemia & $21(30 \%)$ \\
\hline Doenças associadas n(\%) & $22(31,4 \%)$ \\
\hline DPOC & $9(12,9 \%)$ \\
\hline Asma & $2(2,85 \%)$ \\
\hline Tempo de cirurgia $(\min ) *$ & $303,9 \pm 65,8$ \\
\hline CEC (n,\%) & $49(70 \%)$ \\
\hline Tempo de CEC (min) * & $76,35 \pm 25,54$ \\
\hline \multicolumn{2}{|l|}{ ASA n (\%) } \\
\hline 3 & $54(77,1 \%)$ \\
\hline 4 & $4(5,7 \%)$ \\
\hline Tempo de intubação (min) * & $725,76 \pm 298,53$ \\
\hline Tempo de internação (dias)* & $16,5 \pm 5,65$ \\
\hline
\end{tabular}

Tabela 2. Comportamento das provas de função pulmonar.

\begin{tabular}{lccccc}
\hline FP & Pré-op & $1^{\circ} \mathrm{PO}$ & $2^{\circ} \mathrm{PO}$ & $3^{\circ} \mathrm{PO}$ & $5^{\circ} \mathrm{PO}$ \\
\hline VEF1(L) & $1,83 \pm 0,62 \#$ & $0,56 \pm 0,26 \Omega$ & $0,7 \pm 0,38$ & $0,88 \pm 0,42 \theta$ & $1,02 \pm 0,52 \Omega$ \\
CVF(L) & $2,87 \pm 0,81 \#$ & $0,99 \pm 0,44$ & $1,14 \pm 0,44^{* *}$ & $1,34 \pm 0,49 \pi \mathrm{i}$ & $1,71 \pm 0,64 \gamma$ \\
PFE (L/min) & $151,25 \pm 74,33 \#$ & $57,10 \pm 25,84 \beta$ & $72,63 \pm 35,71 ¥ \beta$ & $94,15 \pm 45,9 \lambda$ & $111,19 \pm 63,34^{*}$ \\
Vimáx (mL) & $2360,8 \pm 854,2 \#$ & $638,8 \pm 299,46 \omega$ & $852,6 \pm 453,25 \omega$ & $1143,2 \pm 604,52 \omega$ & $1350,1 \pm 683,44$ \\
\hline
\end{tabular}

FP - função pulmonar; pré-op - pré-operatório; PO - pós-operatório; VEF - volume expiratório forçado no primeiro segundo; CVF capacidade vital forçada; PFE - pico de fluxo expiratório; Vimáx - volume inspiratório máximo; Os valores foram expressos em média e desvio padrão; Teste de ANOVA com Bonferroni; $p<0,05$.

\# representa p<0,01 na comparação entre as medidas do pré-operatório em relação aos dias de pós-operatório.

*representa $p<0,007$ na comparação do PFE do pré-operatório e $5^{\circ} \mathrm{PO}$.

$\Omega$ representa $p<0,01$ na comparação do $V E F_{1}$ entre as medidas $1^{\circ} \mathrm{PO}$ em relação ao $2^{\circ}, 3^{\circ}$ e $5^{\circ} \mathrm{PO} ; 2^{\circ} \mathrm{PO}$ em relação ao $5^{\circ} \mathrm{PO} ; 3^{\circ} \mathrm{PO}$ em relação ao $5^{\circ} \mathrm{PO}$.

$\theta$ representa $p<0,03$ na comparação do VEF1 entre as medidas do $2^{\circ} \mathrm{PO}$ e $3^{\circ} \mathrm{PO}$.

** representa $\mathrm{p}=0,584$ na comparação da CVF entre as medidas do $1^{\circ} \mathrm{PO}$ e $2^{\circ} \mathrm{PO}$.

$\pi$ representa $p<0,02$ na comparação da CVF entre as medidas do $1^{\circ} \mathrm{PO}$ e $3^{\circ} \mathrm{PO}$.

$\gamma$ representa $\mathrm{p}<0,01$ na comparação da CVF entre as medidas do $1^{\circ} \mathrm{PO}$ e $5^{\circ} \mathrm{PO} ; 2^{\circ} \mathrm{PO}$ em relação ao $5^{\circ} \mathrm{PO} ; 3^{\circ} \mathrm{PO}$ em relação ao $5^{\circ} \mathrm{PO}$.

i representa $p=0,081$ na comparação da CVF entre as medidas do $2^{\circ} \mathrm{PO}$ e $3^{\circ} \mathrm{PO}$.

$¥$ representa $p<0,03$ na comparação do PFE entre as medidas do $1^{\circ} \mathrm{PO}$ e $2^{\circ} \mathrm{PO}$.

$\beta$ representa $p<0,01$ na comparação do PFE entre as medidas do $1^{\circ} \mathrm{PO}$ em relação ao $3^{\circ} \mathrm{e} 5^{\circ} \mathrm{PO} ; 2^{\circ} \mathrm{PO}$ em relação ao $3^{\circ} \mathrm{e} 5^{\circ} \mathrm{PO}$.

$\lambda$ representa $p=0,064$ na comparação do PFE entre as medidas do $3^{\circ} \mathrm{PO}$ e $5^{\circ} \mathrm{PO}$.

$\omega$ representa $p<0,01$ na comparação do Vimáx entre as medidas do $1^{\circ} \mathrm{PO}$ em relação ao $2^{\circ}, 3^{\circ}$ e $5^{\circ} \mathrm{PO} ; 2^{\circ} \mathrm{PO}$ em relação ao $3^{\circ}$ e $5^{\circ} \mathrm{PO}$; $3^{\circ} \mathrm{PO}$ em relação ao $5^{\circ} \mathrm{PO}$ 
Os valores percentuais das variáveis $\mathrm{VEF}_{1}$ e CVF apresentaram comportamento semelhante ao dos valores absolutos (Tabela 3). Os valores do pré-operatório do $\mathrm{VEF}_{1}$ e da CVF quando comparados aos dias de pós-operatório apresentaram diferença significativa, $\operatorname{com} P<0,01$. O valor do $1^{\circ} \mathrm{PO}$ do $\mathrm{VEF}_{1}$ comparado aos valores do $2^{\circ}, 3^{\circ}$ e $5^{\circ} \mathrm{PO}$ apresentou diferença significativa com $P<0,01$.

Na comparação do $2^{\circ} \mathrm{PO}$ em relação ao $3^{\circ}$ do $\mathrm{VEF}_{1}$, houve diferença significativa com $P<0,05$, no entanto, quando comparado ao $5^{\circ}$ pós-operatório foi encontrada diferença estatística com $P<0,01$. O valor do $3^{\circ} \mathrm{PO}$ comparado ao $5^{\circ}$ do $\mathrm{VEF}_{1}$ apresentou diferença estatística $\operatorname{com} P<0,02$.

Quando comparado o $1^{\circ} \mathrm{PO}$ da CVF ao $3^{\circ}$ e $5^{\circ}$ pósoperatório, houve diferença significativa com $P<0,04$ e $P<0,01$, respectivamente. A comparação com $2^{\circ} \mathrm{PO}$ não apresentou diferença estatística $\operatorname{com} P=0,928$. Ao comparar o $2^{\circ} \mathrm{PO}$ com o $3^{\circ}$ não foi encontrada diferença significativa com $P=0,196$, porém ao comparar com o $5^{\circ} \mathrm{PO}$ apresentou diferença significativa $P<0,01$.

Em relação à dor, trinta e três pacientes não apresentaram este sintoma durante o período pós-operatório. O local doloroso mais indicado foi a região do esterno e a região da coluna torácica (T10), segundo mais citado.
No $1^{\circ} \mathrm{PO}$, a graduação da dor apresentou mediana de 5 $(2,75-8,0)$, no $2^{\circ} \mathrm{PO}$, este valor foi de $5(2,0-7,0)$, enquanto que no $3^{\circ}$ e $5^{\circ} \mathrm{PO}$, os valores foram $3(0,5-6,0)$ e $3(0-6,0)$, respectivamente. Ao compararmos o valor do $1^{\circ} \mathrm{PO}$ com o $2^{\circ} \mathrm{PO}$, não foi encontrada diferença estatística $(P=0,726)$, porém ao compararmos com o $3^{\circ}$ e $5^{\circ} \mathrm{PO}$ foi observada diferença estatística $(\mathrm{P}<0,01)$.

$\mathrm{Na}$ comparação do $2^{\circ} \mathrm{PO}$ com o $3^{\circ}$, foi encontrada diferença estatística com $P<0,003$, no entanto, ao compararmos com o $5^{\circ} \mathrm{PO}$, não se observou diferença estatística $(P=0,192)$. O $3^{\circ} \mathrm{PO}$, quando comparado ao $5^{\circ}$ $\mathrm{PO}$, não apresentou diferença estatística $(P=0,827)$.

Quando correlacionada a função pulmonar com a dor, verificou-se que houve correlação negativa significativa para as variáveis $\mathrm{VEF}_{1}$, PFE e Vimáx no $1^{\circ}$ pós-operatório. Essa correlação está demonstrada na Tabela 4.

Ao relacionarmos o tempo de internação hospitalar com as variáveis de função pulmonar e a dor, observou-se correlação significativa para os valores pré-operatórios do $\mathrm{VEF}_{1}$ e Vimáx e os valores do $5^{\circ} \mathrm{PO}$ para o PFE e Vimáx (Figura 2). A dor não apresentou correlação com o tempo de internação hospitalar. Os resultados dessa correlação são apresentados na Tabela 5.

Tabela 3. Comportamento das variáveis VEF1 e CVF em valores porcentuais.

\begin{tabular}{lccccc}
\hline FP & Pré-op & $1^{\circ} \mathrm{PO}$ & $2^{\circ} \mathrm{PO}$ & $3^{\circ} \mathrm{PO}$ & $5^{\circ} \mathrm{PO}$ \\
\hline $\mathrm{VEF}_{1}(\%)$ & $63,69 \pm 16,92$ & $18,52 \pm 7,56$ & $22,87 \pm 10,85$ & $28,97 \pm 11,56$ & $33,73 \pm 13,76$ \\
$\mathrm{CVF}(\%)$ & $75,91 \pm 14,13$ & $26,69 \pm 11,43$ & $30,31 \pm 11$ & $35,71 \pm 11,76$ & $45,77 \pm 15,64$ \\
\hline
\end{tabular}

FP - função pulmonar; pré-op - pré-operatório; $P O$ - pós-operatório; $V E F_{1}$ - volume expiratório forçado no primeiro segundo; CVF - capacidade vital forçada; Valores em porcentuais calculados pelo previsto de acordo com as Diretrizes para testes de função pulmonar; Os valores foram expressos em média e desvio padrão; Teste de ANOVA com Bonferroni; $P<0,05$

Tabela 4. Correlação de dor com provas de função pulmonar.

\begin{tabular}{lllllcc}
\hline & \multicolumn{2}{c}{$1^{\circ} \mathrm{PO}$} & \multicolumn{2}{c}{$5^{\circ} \mathrm{PO}$} & \multicolumn{1}{c}{$\Delta$} \\
\hline Variáveis & $\mathrm{r}$ & $P$ & $\mathrm{r}$ & $P$ & $\mathrm{r}$ & $P$ \\
Dor e VEF $_{1}(\mathrm{~L})$ & $-0,262$ & 0,056 & $-0,187$ & 0,229 & $-0,084$ & 0,632 \\
Dor e VEF $_{1}(\%)^{* *}$ & $-0,271$ & $0,047^{*}$ & $-0,119$ & 0,448 & $-0,078$ & 0,657 \\
Dor e CVF (L) & $-0,75$ & 0,590 & $-0,168$ & 0,282 & $-0,026$ & 0,881 \\
Dor e CVF (\%) ** & 0,32 & 0,817 & 0,08 & 0,960 & $-0,021$ & 0,904 \\
Dor e PFE (L/min) & $-0,357$ & $0,008^{*}$ & $-0,198$ & 0,203 & $-0,033$ & 0,851 \\
Dor e Vimáx (mL) & $-0,293$ & $0,032^{*}$ & $-0,234$ & 0,127 & $-0,077$ & 0,654 \\
\hline
\end{tabular}

PO-pós-operatório; $V E F_{1}$ - volume expiratório forçado no primeiro segundo; CVF - capacidade vital forçada; PFE - pico de fluxo expiratório; Vimáx - volume inspiratório máximo; **Valores em percentuais calculados pelo previsto de acordo com as Diretrizes para testes de função pulmonar; $\Delta=$ média do $1^{\circ} \mathrm{PO}-$ média do $5^{\circ}$ PO; Correlação de Spearman; *P<0,05 
Tabela 5. Correlação do tempo de internação com as variáveis de dor e de função pulmonar durante o período do estudo.

\begin{tabular}{lcccccccc}
\hline & \multicolumn{2}{c}{ PRÉ -OP } & \multicolumn{2}{c}{$1^{\circ} \mathrm{PO}$} & \multicolumn{2}{c}{$5^{\circ} \mathrm{PO}$} & \multicolumn{2}{c}{$\Delta$} \\
\hline Variáveis & $\mathrm{r}$ & $P$ & $\mathrm{r}$ & $P$ & $\mathrm{r}$ & $P$ & $\mathrm{r}$ & $P$ \\
TI e Dor & --------- & $-0,064$ & 0,614 & $-0,080$ & 0,598 & $-0,057$ & $0,708 \omega$ \\
TI e VEF $_{1}(\mathrm{~L})$ & $-0,336$ & $0,04^{*}$ & $-0,131$ & 0,324 & $-0,212$ & 0,095 & 0,146 & 0,252 \\
TI e VEF $_{1}(\%) * *$ & $-0,110$ & 0,365 & $-0,025$ & 0,849 & $-0,057$ & 0,660 & 0,020 & 0,873 \\
TI e CVF (L) & $-0,218$ & 0,070 & 0,147 & 0,266 & $-0,125$ & 0,330 & 0,105 & 0,414 \\
TI e CVF (\%) ** & $-0,047$ & 0,699 & 0,283 & $0,30 *$ & 0,051 & 0,690 & 0,075 & 0,558 \\
TI e PFE (L/min) & $-0,196$ & 0,104 & $-0,144$ & 0,278 & $-0,313$ & $0,12^{*}$ & $-0,023$ & 0,856 \\
TI e Vimáx (mL) & $-0,251$ & $0,036^{*}$ & $-0,193$ & 0,143 & $-0,298$ & $0,16^{*}$ & 0,062 & 0,622 \\
\hline
\end{tabular}

pré- op-pré-operatório; $\mathrm{PO}$ - pós-operatório; TI-tempo de internação hospitalar; $V E F_{1}$ - volume expiratório forçado no primeiro segundo; CVF - capacidade vital forçada; PFE - pico de fluxo expiratório; Vimáx - volume inspiratório máximo; **Valores em percentuais calculados pelo previsto de acordo com as Diretrizes para testes de função pulmonar; $\omega \Delta=$ média do $1^{\circ} \mathrm{PO}$ - média do $5^{\circ} \mathrm{PO} ; \Delta=$ média do $1^{\circ} \mathrm{PO}-$ média do $5^{\circ} \mathrm{PO}$; Correlação de Spearman; *P<0,05.

O tempo de intubação apresentou correlação significativa com as variáveis de função pulmonar. Para os valores pré-operatórios somente o valor absoluto do $\mathrm{VEF}_{1}$ apresentou correlação negativa significativa $(r=-0,237 \mathrm{e}$ $P<0,049)$. No $1^{\circ} \mathrm{PO}$, foi encontrada correlação negativa significativa para as variáveis $\mathrm{VEF}_{1}$, PFE e Vimáx $(\mathrm{r}=-0365$ e $P<0,005 ; \mathrm{r}=-0366$ e $P<0,004 ; \mathrm{r}=-0,310$ e $P<0,017$, respectivamente), no $5^{\circ} \mathrm{PO}$ foi observada correlação negativa significativa para as mesmas variáveis do $1^{\circ} \mathrm{PO}(\mathrm{r}$ $=-0,326$ e $P<0,009 ; \mathrm{r}=-0,364$ e $P<0,03 ; \mathrm{r}=-0,331$ e $P<0,007$, respectivamente).

Ao relacionar o tempo de intubação com a variação, valor do pré-operatório diminuído do valor do $5^{\circ} \mathrm{PO}$ para as variáveis de função pulmonar, não foi encontrada correlação significativa. A dor não apresentou correlação significativa com o tempo de ventilação mecânica para os três momentos avaliados, $1^{\circ} \mathrm{PO}(\mathrm{r}=0,146$ e $P=0,245), 5^{\circ} \mathrm{PO}$ ( $\mathrm{r}=0,272$ e $P=0,067)$ e a variação, valor do $1^{\circ} \mathrm{PO}$ diminuído do valor do $5^{\circ} \mathrm{PO}$, (r=0,066 e $\left.P=0,667\right)$.

Apesar do baixo número de pacientes que realizaram a cirurgia combinada, encontrou-se comparação significativa com as variáveis de função pulmonar e dor. Para os valores do $1^{\circ} \mathrm{PO}$, somente na comparação com o PFE foi encontrada diferença estatística com $P<0,05$, para o $5^{\circ} \mathrm{PO}$ foi encontrada diferença estatística com a dor $(P<0,008)$, o $\operatorname{VEF}_{1}(P<0,005)$, a CVF $(P<0,001)$, a CVF percentual $(P<0,014)$, o PFE $(P<0,044)$ e o Vimáx $(P<0,005)$ e para a variação, das variáveis de função pulmonar, do valor percentual do $\mathrm{VEF}_{1}$ $(P<0,013)$ e da CVF $(P<0,039)$.

Quando relacionada a dor com as características dos indivíduos, não houve significância, assim como com as características cirúrgicas. O tempo de analgesia não teve diferença estatística quando comparado com a dor entre os dias de PO.

\section{DISCUSSÃO}

Neste estudo, observou-se importante prejuízo na função pulmonar no PO evidenciado pela redução significativa $(P<0,01)$ quando comparado ao período préoperatório. Essas alterações na função pulmonar são um frequente achado na literatura após a cirurgia cardíaca $[15,16]$, sendo uma complicação bem conhecida, mas suas causas ainda estão sendo exploradas.

A CEC é considerada uma das causas responsáveis pelos danos pulmonares, devido ao aumento da resistência da via aérea e disfunção respiratória [17,18]. O dreno de tórax também traz alteração significativa do $\mathrm{VEF}_{1}$ e da CVF. Guizilini et al. [19] indicaram como sendo pior o dreno intercostal quando comparado ao dreno mediastinal, pela fricção provocada durante o movimento respiratório.

A própria incisão também é questionada por Lichtenberg et al. [4], mostrando que uma mini-incisão gera menor prejuízo à função pulmonar do que a esternotomia mediana, com menor intensidade de dor, tornando possível uma mobilização precoce, graças à estabilidade esternal. Porém, não podemos afirmar esta relação no presente estudo, pois os objetivos não abrangem este tema.

Conforme demonstrado neste estudo, há ainda uma significativa diminuição dos volumes e capacidades pulmonares no quinto dia de pós-operatório. Morsch et al. [15] e Renault et al. [16] também observaram prejuízo significativo da função pulmonar, não se restabelecendo completamente até o sétimo dia de pós-operatório.

No presente estudo, nós avaliamos a dor a partir da EAV, que é uma escala subjetiva e que possui graduação de 0 a 10. Para análise do comportamento da dor no período pós-operatório, os seus valores foram expressos em mediana e percentis 25 e 75 . O período de pós-operatório imediato 
( $\left.1^{\circ} \mathrm{PO}\right)$ apresentou maior grau de intensidade do que o período tardio $\left(5^{\circ} \mathrm{PO}\right)$, evidenciado pela comparação significativa com $P<0,01$. Estes achados assemelham-se com os encontrados na literatura, no entanto poucos estudos avaliam a dor no pós-operatório de cirurgia cardíaca [20].

Ainda permanecem controvérsias em relação à forma descritiva dessa variável, pois os trabalhos que utilizam esta ferramenta descrevem-na como média e desvio padrão, entretanto na Bioestatística é classificada como uma variável qualitativa, sendo a forma adequada de sua apresentação em mediana e percentis 25 e 75, o que tornou difícil a discussão desse achado, em razão desse fato.

A dor localizou-se na região da esternotomia até o $5^{\circ}$ PO, diferindo dos achados encontrados por Giacomazzi et al. [20], nos quais esta se localizou na região da esternotomia até o $3^{\circ} \mathrm{PO}$, passando para o membro inferior associada à presença da safenectomia. Essa variação na localização e na intensidade da dor pode ocorrer porque os estímulos nociceptivos iniciais diminuem com o passar do tempo (pela distância do procedimento cirúrgico, instabilidade esternal e uso de drenos, por exemplo) [11].

A dor apresentou correlação negativa significativa com as variáveis de função pulmonar ( $\mathrm{VEF}_{1}$, PFE e Vimáx), confirmando estudos prévios sobre os danos da função pulmonar no pós-operatório de cirurgia cardíaca [20]. Este achado confirma a alteração da função pulmonar também pela dor. A presença de dreno de tórax, principalmente intercostal, que perfura a pleura intercostal e músculos intercostais [19], limitando a inspiração, é uma causa.

Também o uso da ATIE pode influenciar a função pulmonar. Isso por representar um trauma cirúrgico adicional e diminuir o aporte sanguíneo para a musculatura intercostal, reduzindo a força muscular ventilatória [21]. Entretanto, em nosso estudo não foi avaliada a força muscular respiratória, pois é contra-indicado no período de pós-operatório imediato pelas Diretrizes para Testes de Função Pulmonar [13].

Como demonstrado no presente estudo, foi encontrada uma correlação negativa significativa do tempo de internação quando comparado com os parâmetros de função pulmonar, confirmando que a cirurgia cardíaca via esternotomia possibilita maior prejuízo respiratório, devido à fricção do esterno pela instabilidade do tórax superior, prolongando o período de internação destes pacientes e elevando o custo hospitalar [21].

O tempo de ventilação mecânica apresentou correlação negativa significativa com os parâmetros de função pulmonar. O uso da ventilação mecânica tem sido identificado como um dos principais fatores de lesão pulmonar em adultos submetidos à cirurgia cardíaca. Estudos realizados com pacientes ventilados mecanicamente demonstram alterações do líquido extravascular pulmonar, na permeabilidade capilar, na produção de mediadores inflamatórios e no desenvolvimento de necrose celular [22,23].

Assim como o tempo de ventilação mecânica (VM) demonstrou ser um fator desencadeador do prejuízo na função pulmonar, o tipo de cirurgia também foi responsável por este declínio. Segundo Renault et al. [18], o trauma cirúrgico pode alterar as propriedades contráteis do diafragma e resultar na incoordenação da caixa torácica, podendo causar fadiga nos músculos respiratórios.

Em nosso estudo observou-se diferença significativa quando comparadas as provas de função pulmonar com a cirurgia combinada. Na literatura há evidências de que o uso da ATIE possa ser um fator responsável pelo prejuízo na função pulmonar. Mueller et al. [21] relatam que o impacto da ATIE é moderado no pós-operatório baseado no maior trauma cirúrgico que este enxerto exige, porém este estudo não realiza provas de função pulmonar.

Ao comparar a dor com o tipo de cirurgia encontramos diferença estatística significativa para a cirurgia combinada. Diferindo dos achados encontrados por Mueller et al. [1], que não apresentaram diferença significativa nas características da dor quando comparados diferentes tipos de cirurgia, mesmo em procedimentos mais profundos, com maior estímulo nociceptivo como cirurgias valvares, mas o autor não descreve se foram incluídos pacientes que fizeram uso de ATIE. No entanto, nesta pesquisa, não encontramos relação entre uso da ATIE e intensidade de dor, pois dos 70 indivíduos estudados, somente 13 utilizaram esse enxerto e nenhuma das cirurgias combinada utilizou-o na revascularização do miocárdio.

No presente estudo, não observamos relações significantes entre as características dos indivíduos (idade, sexo e IMC, por exemplo) e do procedimento cirúrgico. Indivíduos que apresentaram um IMC maior de $30 \mathrm{~kg} / \mathrm{m}^{2}$ referiram mais dor, possivelmente pelo fato da dificuldade no manejo analgésico adequado destes [1]. Os achados deste estudo não podem confirmar essa hipótese, pois apenas seis pacientes apresentavam este valor de IMC.

O nosso estudo apresentou algumas limitações. Dentre elas, o grande número de perdas, sendo as cirurgias canceladas ou antecipadas as mais frequentes (Figura 1), isso pode ter ocorrido em razão do fechamento do bloco cirúrgico, pois a Instituição estava analisando melhores condições de atendimento do Sistema Único de Saúde. A não realização de uma avaliação radiológica, que poderia evidenciar complicações pulmonares de diferentes proporções, afetando os valores de função pulmonar. Os testes apresentam uma limitação, pois seus resultados dependem da compreensão das manobras e da vontade do pacientes em realizá-las. O recurso para avaliação do volume inspiratório máximo não é mais específico, porém de fácil reprodutibilidade e baixo custo.

Trabalhos prévios sugerem que pacientes mais 
preparados e colaborativos com a equipe de reabilitação podem identificar precocemente a dor [23]. Como a dor é um sintoma frequente no pós-operatório de cirurgia cardíaca, os profissionais da equipe de reabilitação também devem receber educação sobre este tema, visando a sua identificação e manejo precoce.

\section{CONCLUSÃO}

No presente estudo, observou-se prejuízo significativo da função pulmonar, que não retornou até o $5^{\circ}$ PO. Essa alteração pode ser percebida melhor com a relação significativa da dor com os parâmetros de função pulmonar. A dor localizou-se na região da esternotomia, sendo uma queixa que persistiu durante todo o período do estudo. A função pulmonar apresentou correlação negativa significativa com o tempo de ventilação mecânica e o tempo de internação hospitalar, podendo ter sido determinado pela associação da função pulmonar pré-operatória com o trauma cirúrgico. Observou-se diferença significativa entre a cirurgia combinada e os parâmetros de função pulmonar e a dor, sendo explicado pela grande manipulação cirúrgica acarretando essas alterações no período pós-operatório. Não houve correlação entre dor e as características dos indivíduos, do procedimento cirúrgico e tempo de internação hospitalar.

\section{AGRADECIMENTOS}

Gostaríamos de agradecer às Equipes da Unidade de Cardiologia da Policlínica Santa Clara e da Unidade de Terapia Intensiva (UTI) Cirúrgica do Hospital São Francisco do Complexo Hospitalar Santa Casa de Porto Alegre, Rio Grande do Sul, pelo empenho e colaboração para realização do presente estudo.

\section{REFERÊNCIAS}

1. Mueller XM, Tinguely F, Tevaearai HT, Revelly JP, Chioléro $\mathrm{R}$, von Segesser LK. Pain location, distribution, and intensity after cardiac surgery. Chest. 2000;118(2):391-6.

2. Magnano D, Montalbano R, Lamarra M, Ferri F, Lorini L, Clarizia S, et al. Ineffectiveness of local wound anesthesia to reduce postoperative pain after median sternotomy. J Card Surg. 2005;20(4):314-8.
3. Cimen S, Ozkul V, Ketenci B, Yurtseven N, Günay R, Ketenci $\mathrm{B}$, et al. Daily comparison of respiratory functions between on-pump and off-pump patients undergoing CABG. Eur J Cardiothorac Surg. 2003;23(4):589-94.

4. Lichtenberg A, Hagl C, Harringer W, Klima U, Haverich A. Effects of minimal invasive coronary artery bypass on pulmonary function and postoperative pain. Ann Thorac Surg. 2000;70(2):461-5.

5. Mueller XM, Tinguely F, Tevaearai HT, Ravussin P, Stumpe $\mathrm{F}$, von Segesser LK. Impact of duration of chest tube drainage on pain after cardiac surgery. Eur J Cardiothorac Surg. 2000;18(5):570-4.

6. Westerdahl E, Lindmark B, Bryngelsson I, Tenling A. Pulmonary function 4 months after coronary artery bypass graft surgery. Respir Med. 2003;97(4):317-22.

7. Taggart DP. Respiratory dysfunction after cardiac surgery: effects of avoiding cardiopulmonary bypass and the use of bilateral internal mammary arteries. Eur J Cardiothorac Surg. 2000;18(1):31-7.

8. Erb J, Orr E, Mercer CD, Gilron I. Interactions between pulmonary performance and movement-evoked pain in the immediate postsurgical period: implications for perioperative research and treatment. Reg Anesth Pain Med. 2008;33(4):312-9.

9. Bonnet F, Marret E. Postoperative pain management and outcome after surgery. Best Pract Res Clin Anaesthesiol. 2007;21(1):99-107.

10. Almeida PLBM. Impacto da reabilitação cardiopulmonar intrahospitalar pré e pós-operatória em ex-tabagistas submetidos à cirurgia de revascularização miocárdica - um ensaio clínico randomizado [Dissertação de Mestrado]. Porto Alegre: Universidade Federal do Rio Grande do Sul;2007. [citado 2008 Set 26]. Disponível em: URI: http:/hdl.handle.net/10183/14035.

11. Watt-Watson J, Stevens B, Katz J, Costello J, Reid GJ, David T. Impact of preoperative education on pain outcomes after coronary artery bypass graft surgery. Pain. 2004;109(1-2):73-85.

12. Romanini W, Muller AP, Carvalho KAT, Olandoski M, FariaNeto JR, Mendes FL, et al. Os efeitos da pressão positiva intermitente e do incentivador respiratório no pós-operatório de revascularização miocárdica. Arq Bras Cardiol. 2007;89(2):105-10.

13. Müller AP, Olandoski M, Macedo R, Costantini C, GuaritaSouza LC. Comparative study between intermittent (Müller Reanimator) and continuous positive airway pressure in the postoperative period of coronary artery bypass grafting. Arq Bras Cardiol. 2006;86(3):232-9.

14. Pereira CAC, Jansen JM, Barreto SSM, Marinho J, Sulmonett N, Dias RM. Espirometria. In: Diretrizes para testes de função pulmonar. J Pneumol. 2002;28:S1-S82. 
15. Morsch KT, Leguisamo CP, Camargo MD, Coronel CC, Mattos W, Ortiz LDN, et al. Perfil ventilatório dos pacientes submetidos a cirurgia de revascularização do miocárdio. Rev Bras Cir Cardiovasc. 2009;24(2):180-7.

16. Renault JA, Costa-Val R, Rossetti MB, Houri Neto M. Comparação entre exercícios de respiração profunda e espirometria de incentivo no pós-operatório de cirurgia de revascularização do miocárdio. Rev Bras Cir Cardiovasc. 2009;24(2):165-72

17. Silva AMRP, Campagnucci VP, Pereira WL, Rosa RF, Franken RA, Gandra SMA, et al. Revascularização do miocárdio sem circulação extracorpórea em idosos: análise da morbidade e mortalidade. Rev Bras Cir Cardiovasc. 2008;23(1):40-5.

18. Renault JA, Costa-Val R, Rossetti MB. Fisioterapia respiratória na disfunção pulmonar pós-cirurgia cardíaca. Rev Bras Cir Cardiovasc. 2008;23(4):562-9.

19. Guizilini S, Gomes WJ, Faresin SM, Carvalho ACC, Jaramillo JI, Alves FA, et al. Efeitos do local de inserção do dreno pleural na função pulmonar no pós-operatório de cirurgia de revascularização do miocárdio. Rev Bras Cir Cardiovasc. 2004;19(1):47-54.

20. Giacomazzi CM, Lagni VB, Monteiro MB. A dor pósoperatória como contribuinte do prejuízo na função pulmonar em pacientes submetidos à cirurgia cardíaca. Rev Bras Cir Cardiovasc. 2006;21(4):386-92.

21. Mueller XM, Tinguely F, Tevaearai HT, Revelly JP, Chioléro $\mathrm{R}$, von Segesser LK. Pain pattern and left internal mammary artery grafting. Ann Thorac Surg. 2000;70(6):2045-9.

22. Wrigge H, Uhlig U, Baumgarten G, Menzenbach J, Zinserling $\mathrm{J}$, Ernst M, et al. Mechanical ventilation strategies and inflammatory responses to cardiac surgery: a prospective randomized clinical trial. Intensive Care Med. 2005;31(10):1379-87.

23. Wrigge $H$, Uhlig U, Zinserling J, Behrends-Callsen E, Ottersbach G, Fischer M, et al. The effects of different ventilatory settings on pulmonary and systemic inflammatory responses during major surgery. Anesth Analg. 2004;98(3):775-81. 